\title{
Plasma rico em plaquetas (PRP): classificação, mecanismos de ação e métodos de obtenção
}

\author{
Platelet-rich plasma (PRP): classification, mechanisms of action and methods of \\ production
}
Plasma rico en plaquetas (PRP): clasificación, mecanismos de acción y métodos de producción

Kurt Vinicius Menezes Schneider ${ }^{1 \star}$, Ronald Bispo Barreto da Silva1.

\begin{abstract}
RESUMO
Objetivo: Esclarecer a comunidade da área da saúde na compreensão da dificuldade de padronização de um produto autólogo com grande capacidade de aplicação na medicina regenerativa e evidenciar sua classificação, mecanismo de ação e produção. Revisão bibliográfica: Empregado inicialmente na década de 1970 para prevenir hemorragia, o plasma rico em plaquetas (PRP) tem ampliado o seu campo de atuação devido à sua capacidade em medicina regenerativa. Utiliza o sangue do próprio paciente e consiste em uma fração do plasma com uma concentração de plaquetas maior do que o sangue original. O princípio de atuação é baseado principalmente na liberação de fatores de crescimento pelas plaquetas quando ativadas. Pode ser preparado de diversas maneiras e envolve centrifugação. Pode ter concentrações variadas de plaquetas e leucócitos. Devido à sua variabilidade na preparação e apresentação, é difícil interpretar os dados disponíveis na literatura. Várias maneiras de classificação foram propostas para ajudar na sistematização e comparação dos estudos. Considerações finais: O PRP é um produto que tem apresentado bons resultados em várias áreas da medicina regenerativa. O conhecimento do mecanismo de ação, métodos de preparo e classificação auxilia os profissionais de saúde a melhor entender essa nova terapia e incentiva a sua utilização.
\end{abstract}

Palavras-chave: Plasma rico em plaquetas, Ativação plaquetária, Medicina regenerativa.

\begin{abstract}
Objective: To clarify the health community in understanding the difficulty of standardizing an autologous product with great capacity for application in regenerative medicine and highlight its classification, mechanism of action and production. Literature review: Initially employed in the 1970s to prevent hemorrhage, the platelet-rich plasma (PRP) has expanded its field of action due to its capacity in regenerative medicine. It uses the patient's own blood and consists of a fraction of the plasma with a higher platelet concentration than the original blood. The principle of action is based mainly on the release of growth factors by platelets when activated. It can be prepared in several ways and involves centrifugation. It may have varying concentrations of platelets and leukocytes. Due to its variability in preparation and presentation, it is difficult to interpret the data available in the literature. Various ways of classification have been proposed to help systematize and compare studies. Final Considerations: PRP is a product that has shown good results in several areas of regenerative medicine. Knowledge of the mechanism of action, methods of preparation and classification helps health professionals to better understand this new therapy and encourages its use.
\end{abstract}

Key words: Platelet-rich plasma, Platelet activation, Regenerative medicine.

${ }^{1}$ Universidade Tiradentes (UNIT), Aracaju - SE. *E-mail: schneiderkurt07@gmail.com

SUBMETIDO EM: 2/2020

ACEITO EM: 3/2020

PUBLICADO EM: 5/2020

REAS/EJCH | Vol.Sup.n.47 | e3184 | DOI: https://doi.org/10.25248/reas.e3184.2020 Página 1 de 8 


\section{RESUMEN}

Objetivo: Aclarar a la comunidad de la salud para comprender la dificultad de estandarizar un producto autólogo con gran capacidad de aplicación en medicina regenerativa y resaltar su clasificación, mecanismo de acción y producción. Revisión bibliográfica: Inicialmente empleado en la década de 1970 para prevenir la hemorragia, el plasma rico en plaquetas (PRP) ha ampliado su campo de acción debido a su capacidad en medicina regenerativa. Utiliza la propia sangre del paciente y consiste en una fracción del plasma con una concentración de plaquetas más alta que la sangre original. El principio de acción se basa principalmente en la liberación de factores de crecimiento por las plaquetas cuando se activa. Se puede preparar de varias maneras e implica centrifugación. Puede tener concentraciones variables de plaquetas y leucocitos. Debido a su variabilidad en la preparación y presentación, es difícil interpretar los datos disponibles en la literatura. Se han propuesto varias formas de clasificación para ayudar a sistematizar y comparar estudios. Consideraciones finales: PRP es un producto que ha mostrado buenos resultados en varias áreas de la medicina regenerativa. El conocimiento del mecanismo de acción, métodos de preparación y clasificación ayuda a los profesionales de la salud a comprender mejor esta nueva terapia y fomenta su uso.

Palabras clave: Plasma rico en plaquetas, Activación plaquetaria, Medicina regenerativa.

\section{INTRODUÇÃO}

O Plasma Rico em Plaquetas (PRP) é definido como um produto biológico autólogo derivado do sangue do paciente e do qual, após um processo de centrifugação, é obtida uma fração plasmática com uma concentração de plaquetas maior que a do sangue circulante (CHICHARRO-ALCÁNTARA D, et al., 2018). Ele contém substâncias bioativas, incluindo fatores de crescimento, que são capazes de acelerar o processo de regeneração dos tecidos (ARAKI J, et al., 2012).

O desenvolvimento do conceito de PRP teve início da década de 1970, no campo da hematologia das transfusões de sangue, onde foi usado para tratar e prevenir hemorragia devido a trombopenia (ALVES R e GRIMALT R, 2018). Ainda hoje os concentrados de plaquetas (CP) são indicados para transfusões em pacientes com trombopenia. Os concentrados de plaquetas são considerados PRP se for um produto de sangue autólogo (sangue do próprio paciente), mas nas transfusões são utilizados principalmente concentrados de plaquetas de doadores. Segundo dados do Ministério da Saúde, a média de transfusão de sangue no Brasil, de 2014 a 2016, foi de 3.173.524 procedimentos realizados. Em 2016, dos 2.840 .988 procedimentos, 12,59\% foram de concentrado de plaquetas (BRASIL, 2018).

Posteriormente o uso do PRP foi expandido para outros campos, principalmente devido à sua capacidade potencial em medicina regenerativa (PIERCE GF, et al., 1991), incluindo cirurgia buco-maxilo-facial (STÄHLI A, et al., 2018) e medicina esportiva (CHARLES MD, et al., 2018).

O plasma rico em plaquetas (PRP) tem sido objeto de centenas de publicações nos últimos anos. Na medicina esportiva e na ortopedia o PRP tem sido de interesse para promover a cura fisiológica acelerada e o retorno à função (BOSWELL SG, et al., 2012). Relatos de seus efeitos nos tecidos, positivos e negativos, têm gerado grande interesse na comunidade ortopédica (DELONG JM, et al., 2012). Tornou-se uma opção de tratamento para as lesões osteomusculares e seu uso é amplamente difundido em países da Europa e EUA. Diferentes destas regiões, no Brasil, o uso não-transfusional do PRP, como produto de terapia avançada para agravos da saúde, ainda não está regulamentado. O Conselho Federal de Medicina (CFM), na sua Resolução 2.128/2015, considera o PRP como procedimento experimental, só podendo ser utilizado em experimentação clínica dentro dos protocolos do sistema CEP/CONEP.

Os resultados clínicos apresentados têm sido melhores que outras terapias injetáveis no tratamento de artrose do joelho (SHEN L, et al., 2017) e das tendinopatias do ombro. Ao comparar com o uso de corticoides, o PRP tem mostrado resultados mais positivos, podendo ser considerado como uma boa alternativa para o tratamento de doenças osteoarticulares, especialmente em pacientes com contraindicação à administração dos esteroides. 
Com o objetivo de contribuir para este estudo foi realizada uma revisão bibliográfica narrativa do conceito de PRP, abrangendo as tentativas de classificação, as dificuldades de comparar os resultados clínicos e experimentais, seus componentes, mecanismos de ação e o seu preparo.

\section{REVISÃO BIBLIOGRÁFICA}

O termo PRP não é utilizado de uma forma homogênea entre os diversos autores da literatura científica mundial, o que dificulta a comparação de resultados entre os diversos estudos de obtenção do PRP e da sua eficácia clínica. Isso ocorre, principalmente, porque nas publicações mais iniciais sobre as aplicações clínicas do PRP, diferentes termos foram usados para o mesmo produto, da mesma forma que diferentes produtos eram descritos sob a mesma nomenclatura (GAUDRIC A, et al., 1995; WHITMAN DH, et al., 1997).

Fatores como variações nas preparações e dosagens, a presença ou ausência de leucócitos, e fatores relacionados ao paciente, como idade, sexo, histórico da doença e histórico de tratamento, entre outros, podem afetar os resultados dos estudos (ZHOU Y, et al., 2015).

Com objetivo de classificar os tipos de PRP e melhor comparar os resultados dos experimentos, os autores começaram a identificar parâmetros diferenciadores entre os diversos tipos de preparados de plaquetas, tais como concentração de plaquetas, níveis de concentração de leucócitos e tipo de ativação (ZHOU Y, et al., 2015). Assim, Delong JM, et al. (2012); Ehrenfest DMD, et al. (2009) identificaram características comuns às diversas técnicas de PRP e propuseram classificações dos diferentes tipos de PRP existentes.

Ehrenfest DMD, et al. (2009) classificaram os diferentes concentrados de plaquetas em quatro categorias, dependendo da densidade de fibrina, conteúdo de leucócitos e grau de padronização do procedimento: plasma rico em plaquetas puro (P-PRP), plasma rico em leucócitos e plaquetas (L-PRP), plasma rico em plaquetas puro e rico fibrina (P-PRF) e rico em plaquetas, leucócitos e fibrinas (L-PRF).

Em 2012, Delong JM, et al. (2012) propuseram a classificação PAW para organizar e comparar resultados na literatura. Este sistema de classificação é baseado em três componentes: a quantidade de plaquetas $(P)$, a maneira pela qual a ativação plaquetária ocorre $(\mathrm{A})$ e a presença ou ausência de leucócitos $(\mathrm{W}=$ White Blood Cells). Este sistema de classificação conseguiu simplificar a complexa tipologia do PRP valorizando mais o conteúdo celular do preparado e menos a metodologia de obtenção do produto.

Outro sistema de classificação proposto é a classificação MARSPILL (M: Method; A: activation, R: red blood cells, S: spin, P: platelets, I: image guidance, L: leukocytes, L: light activation), onde as letras no nome designam a referência aos processos de preparação (por exemplo, $\mathrm{M}=$ manual ou processado de forma automatizada. Nesta classificação os autores destacaram a importância das células mononucleares e incorporaram variáveis não presentes em outras classificações, como a ativação por luz (LANA JFSD, et al., 2017).

\section{Componentes bioquímicos e bioativos do PRP}

Originários do sangue, os diversos tipos de PRP possuem um grande número de componentes e substâncias bioativas, que desempenham funções variadas nos processos de recuperação dos tecidos. Levando-se em consideração a importância para a medicina regenerativa, pode-se destacar os três principais componentes do PRP, que são as plaquetas, o plasma e os leucócitos. Cada um destes componentes possui suas substâncias bioativas (BOSWELL SG, et al., 2012).

\section{Plaquetas}

As plaquetas, principais componentes do PRP, são fragmentos celulares discóides sem núcleo originário de megacariócitos. As plaquetas desempenham um papel importante na hemostasia normal, interrompendo a perda de sangue após lesão vascular. Elas contribuem para diversos processos imunológicos (ALI RA, et al., 2015; MOOJEN DJF, et al., 2008). Na reparação de tecidos e na cicatrização de feridas, as plaquetas desempenham um papel crucial, graças à sua função hemostática, a presença de citocinas e fatores de crescimento (CHICHARRO-ALCÁNTARA D, et al., 2018). 
Elas possuem um grande número de moléculas biologicamente ativas contidas em seus inúmeros grânulos, que são de três tipos: grânulos alfa, grânulos densos e lisossomos, contendo várias proteínas e substâncias bioativas (BOSWELL SG, et al., 2012).

Os grânulos alfa são os mais abundantes, perfazendo aproximadamente $10 \%$ do volume das plaquetas. Eles contêm moléculas bioativas como proteínas adesivas (fibrinogênio e fator von Willebrand), fatores de coagulação ( $\mathrm{V}, \mathrm{XI}, \mathrm{XIII}$ e protrombina), fatores fibrinolíticos (antitrombina, plasmina e plasminogênio), integrinas ( $\alpha 2 b, a 6, \beta 3)$, molécula de adesão celular endotelial de plaquetas (PECAM) e os fatores de crescimento: fator de crescimento derivado de plaquetas (PDGF), fator de crescimento transformador beta 1 (TGF- $\beta 1$ ), fator de crescimento endotelial vascular (VEGF), fator de crescimento fibroblástico básico (bFGF), fator de crescimento do hepatócito (HGF) e fator de crescimento epidermal (EGF) (BLAIR P e FLAUMENHAFT $\mathrm{R}, 2009$ ).

O PDGF é essencial para a proliferação celular, quimiotaxia, diferenciação celular e angiogênese (SCHMIDT MB, et al., 2006). O aumento da proliferação celular é uma das formas do PRP promover a cicatrização de tecidos. Chung R, et al. (2009) comprovaram que o bloqueio de PDGF em um modelo animal de dano na placa de crescimento de roedores resultou na redução da quimiotaxia de células mesenquimais, prejudicando as respostas condrogênicas, osteogênicas e reparadoras teciduais.

Os grânulos densos (delta) contêm principalmente moléculas que estimulam o processo de coagulação, incluindo o cálcio, adenosina difosfato (ADP), adenosina trifosfato (ATP), serotonina e histamina. A serotonina participa nos mecanismos de regulatórios da massa óssea e níveis elevados deste hormônio ou neurotransmissor podem regular negativamente a densidade mineral óssea e aumentar o risco de fraturas (MEHTA SK, et al., 2012).

O terceiro tipo de grânulos são os lisossomos (grânulos lambda), cujo conteúdo é liberado pela ativação das plaquetas. Contém enzimas necessárias no processo de degradação de proteínas (proteases), lipídios (lipases) e carboidratos (glicosidases), que também estão envolvidos na remoção dos detritos do tecido danificado, na remoção dos agentes infecciosos e no próprio processo de coagulação (MENG R, et al., 2015).

A ativação das plaquetas pode ocorrer por moléculas endógenas ou exógenas, podendo participar deste processo o colágeno, fator ativador de plaquetas, serotonina, cálcio, magnésio, tromboxano A2 (TXA2), ADP e trombina (BOSWELL SG, et al., 2012).

\section{Plasma}

O plasma é o componente líquido de cor amarelo presente no sangue em que as células ficam suspensas. O plasma também é parte ativa do PRP. Na sua composição encontram-se proteínas, eletrólitos como cálcio e cloretos, hormônios e outras substâncias que participam no mecanismo de ação do PRP como moléculas de sinalização e de ativação de plaquetas (ALVES R e GRIMALT R, 2018; BOSWELL SG, et al., 2012).

As proteínas plasmáticas envolvidas na hemostasia atuam também na cicatrização tecidual. Depois que o tampão de plaquetas ativadas fornece hemostasia primária no local da lesão, as moléculas de adesão celular como fibronectina, fibrina e vitronectina se movem do plasma para o coágulo, durante a sua maturação. In vitro, essas proteínas demonstraram induzir a quimiotaxia de células-tronco multipotentes através de uma membrana micro-porosa padrão. Isso implica que essas moléculas podem modular a migração celular de fibroblastos, osteoblastos ou outras células regeneradoras no reparo dos tecidos. (THIBAULT MM, et al., 2007; BOSWELL SG, et al., 2012).

Cloreto, sódio, potássio e cálcio são os quatro eletrólitos que são mais abundantes no plasma. Nas funções celular e tecidual, eles são fortemente regulados através de adenosina trifosfatases transmembrana. O cálcio é um importante mensageiro secundário nas células e é cofator de várias reações extracelulares. Em relação à reparação tecidual, a sinalização intracelular do cálcio é necessária para a atividade contrátil dos miofibroblastos (BOSWELL SG, et al., 2012) e também na mediação de sinais mecânicos intracelulares e extracelulares, organizando estruturalmente o citoesqueleto de actina-miosina contrátil e estabelecendo linhas de comunicação intercelular (BOSWELL SG, et al., 2012; FOLLONIER CASTELLA L, et al., 2010). 


\section{Leucócitos}

Os Leucócitos podem ser classificados em cinco grandes grupos: neutrófilos, eosinófilos, basófilos, linfócitos e monócitos (ou macrófagos). Os neutrófilos e os macrófagos são constituídos por células fagocíticas e são essenciais para o processo de cicatrização. Após a hemostasia e coagulação, já na fase inflamatória do processo de cicatrização, neutrófilos e logo em seguida macrófagos migram para a ferida. $O$ trabalho dos neutrófilos é crucial nos primeiros dias após a lesão, porque sua capacidade de fagocitose e secreção de protease elimina as bactérias locais e ajuda a degradar o tecido necrótico (REINKE JM e SORG H, 2012).

Em seguida e concomitantemente com o influxo de neutrófilos, os monócitos circulantes entram na ferida e se diferenciam em macrófagos maduros. Os macrófagos desempenham um papel central e coordenador no reparo tecidual, secretando citocinas e fatores de crescimento que influenciam as atividades dos fibroblastos. Também fagocitam os neutrófilos mortos, desbridam ainda mais a ferida e produzem fatores que estimulam a angiogênese (KOH TJ e DIPIETRO LA, 2011).

No processo de cicatrização dos tecidos os leucócitos produzem vários fatores de crescimento no local da lesão (CIESLIK-BIELECKA A, et al., 2012). Estes dados sugerem que o PRP rico em leucócitos poderia aumentar a disponibilidade do fator de crescimento para tecidos lesionados em comparação com os métodos pobres em leucócitos (DAVIS VL, et al., 2014).

Os leucócitos liberam também muitas proteinases, como serino-proteases e metaloproteinases (MMP), que tem papel fundamental na cicatrização de feridas. As proteinases controlam a resposta inflamatória desativando as células inflamatórias para limitar a lesão dos tecidos circundantes durante a cicatrização de feridas (DAVIS VL, et al., 2014; CALEY MP, et al., 2015).

A concentração ideal de leucócitos no PRP ainda é controversa. Por um lado, os leucócitos são benéficos por estimular a resposta imune contra infecções; promover quimiotaxia, proliferação e diferenciação de células; e induzir produção de matriz extracelular e angiogênese. Devido a isso, os leucócitos contendo PRP (L-PRP) são frequentemente usados para tratar lesões traumáticas, como a cicatrização de lesões dérmicas grandes e infectadas. Por outro lado, os leucócitos também liberam citocinas inflamatórias e espécies reativas de oxigênio, ambas com efeitos prejudiciais sobre os tecidos tratados (ZHOU Y, et al., 2015; BOSWELL SG, et al., 2012). Assim, o PRP sem leucócitos concentrados pode ser mais adequado para o tratamento de lesões da cartilagem articular (XU Z, et al., 2017).

\section{Mecanismo de ação do PRP}

Para agir no local lesado, o PRP é ativado. O termo ativação refere-se ao estímulo dado para que haja a degranulação das plaquetas, causando a liberação dos fatores de crescimento (FCs) e outras substâncias bioativas (CAVALLO C, et al., 2016).

O plasma do PRP contém citocinas, trombina e outros fatores de crescimento com propriedades biológicas e adesivas. O PRP pode ser pré-ativado com trombina e ou cloreto de cálcio antes da utilização. Mas também ele pode ser ativado espontaneamente por ativadores presentes no local a ser tratado. Entre estes ativadores estão o colágeno e trombina endógena (DAVIS VL, et al., 2014).

Com a ativação, dentre as substâncias secretadas, temos: fator de crescimento derivado de plaquetas (PDGF), antagonista do receptor da interleucina-1 (IL-1RA), receptores solúveis do fator de necrose tumoral $\alpha$ (TNF-RI), fator de crescimento transformador $\beta$ (TGF- $\beta$ ), fator plaquetário 4 (PF4), fator de crescimento do endotélio vascular (VEGF), fator de crescimento epidermal (EGF), fator de crescimento semelhante a insulina (IGF), osteocalcina (Oc), osteonectina (On), fibrinogênio, vitronectina, fibronectina e trombospondina-1 (TSP1) (KNOP E, et al., 2016).

Estas substâncias bioativas ajudam a iniciar e aceleram a reparação e regeneração de tecidos. Podem suprimir a liberação de citocinas e limitar a inflamação, interagindo com macrófagos para melhorar a cicatrização (LACCI KM e DARDIK A, 2010). 
Os fatores de crescimento sinalizam proteínas que influenciam o metabolismo de outras células. Cada fator de crescimento (FC) tem mais de um efeito no processo de cicatrização de feridas e age ligando-se a receptores específicos nas membranas celulares das células alvo (CHICHARRO-ALCÁNTARA D, et al., 2018).

O PDGF promove a síntese de colágeno, angiogênese e a proliferação das células ósseas (AHMAD Z, et al., 2012). O TGF- $\beta$ significativamente aumenta e estimula a deposição de matriz extracelular (BHANOT S e ALEX JC, 2002). O TGF- $\beta 1$ também atua como um fator inibidor da degradação da cartilagem, regula e aumenta a expressão dos genes dos inibidores teciduais das metaloproteinases (TIMP-1) (KNOP E, et al., 2016). O aumento da concentração de IL-1 $\beta$ e TNFa no ferimento, atraem maior migração de macrófagos, fibroblastos e aumentam a produção de fibras de colágeno contribuindo para um melhor e mais rápido processo de cicatrização de ferida pós-operatória (MENCHISHEVA Y, et al., 2018).

A presença aumentada de IGF-1, FGF-2, TGFß-1 e PDGF aumentam a proliferação e diferenciação de mioblastos, aumentam o diâmetro e o número das fibras musculares, além de reduzir a formação de fibrose dentro das lesões musculares (BORRIONE P, et al., 2018).

\section{Métodos de obtenção e preparação do PRP}

O PRP é um produto de sangue autólogo (sangue do próprio paciente) que é processado por centrifugação diferencial, para concentrar o máximo número de plaquetas, que é então injetado de volta no paciente no local da lesão ou da patologia. Na centrifugação diferencial a aceleração é ajustada para sedimentar certos constituintes celulares com base na diferença de massa específica (DHURAT R e SUKESH M, 2014).

Até o momento, não há consenso sobre o melhor método de preparação do PRP. O volume de sangue adquirido, concentração de plaquetas, presença de leucócitos e eritrócitos, uso de kit de preparação versus manuseio manual, etapas de centrifugação, métodos de ativação, número e tempo de injeções, e cuidados no procedimento proporcionam grandes variações em formulações e resultados.

De uma maneira geral, pode-se identificar duas técnicas de preparação: técnica aberta e técnica fechada (ALVES R e GRIMALT R, 2018). Na técnica aberta o produto é exposto ao ambiente da área de trabalho e entra em contato com os diferentes materiais utilizados na sua produção. Nesse tipo de processamento devese garantir que o produto não seja contaminado durante o manuseio. Na técnica fechada são utilizados dispositivos disponíveis comercialmente, oficialmente registrados, nos quais o produto não é exposto ao ambiente. A preparação do PRP pela técnica fechada vai depender do tipo de dispositivo escolhido e deve ser feito de acordo com as instruções do fabricante. Existem diferentes sistemas de PRP que facilitam a sua preparação de maneira simples e reprodutível. Todos operam com um pequeno volume de sangue tirado e segue o princípio da centrifugação. O sangue então é centrifugado com centrifugação simples ou dupla, dependendo do dispositivo (ALVES R e GRIMALT R, 2018).

Um dos protocolos de preparação descritos na literatura é o seguinte: O sangue total é obtido por venopunção em tubo com anticoagulantes, para prevenir que as plaquetas sejam ativadas antes da utilização. A primeira centrifugação é realizada e o tubo mostra três camadas: a camada inferior é composta principalmente por hemácias, a camada intermediária é rica em leucócitos e a camada mais superior é composta por plasma rico em plaquetas e alguns leucócitos. Para a preparação do puro PRP (P-PRP) a camada superior e a parte superficial da camada intermediária são transferidas para outro tubo estéril e então é novamente centrifugado. Para a produção do PRP rico em leucócitos (L-PRP) a camada intermediária é transferida toda. Após a segunda centrifugação a porção superior do volume, que é basicamente o PPP (plasma pobre em plaqueta) é removida e a parte restante é homogeneizada para criar o PRP (DHURAT Re SUKESH M, 2014).

\section{Formas de apresentação do PRP}

Os produtos de PRP, normalmente, são bem diferentes uns dos outros. Os diferentes métodos de preparação, os diferentes sistemas comerciais utilizados, se são ricos ou não em leucócitos, se são préativados e muitos outros fatores que afetam o produto final. A concentração dos fatores de crescimento e de 
citocinas catabólicas são influenciadas pela composição celular do PRP incluindo a presença ou não de leucócitos (BOSWELL SG, et al., 2012).

O PRP pode se apresentar na forma líquida ou sólida e cada uma delas possuem propriedades diferentes. A diferença principal é o método de aplicação no local da lesão. O PRP líquido pode ser injetado guiado por ultrassonografia ou por referência topográfica (método palpatório). No entanto, o sólido é aplicado sob visão direta da lesão, por meio de incisão cirúrgica. O PRP sólido apresenta-se como uma malha estruturada de fibrina, a qual é criada utilizando dupla centrifugação. Esta estrutura ou malha de fibrina serve como um reservatório para os fatores de crescimento e permite a liberação gradual destes fatores (BARBER FA, 2018).

O PRP pode ser preparado, congelado e depois retificado novamente antes da injeção ou usado fresco sem congelamento. O congelamento teria a vantagem de disponibilizar maior quantidade para o paciente que precisasse utilizar várias injeções. No entanto a maioria dos estudos utiliza PRP fresco porque parece ser mais eficaz (BENNELL KL, et al., 2017).

\section{CONSIDERAÇÕES FINAIS}

Desde o início de sua utilização na medicina regenerativa e da observação de terapias com resultados positivos, o espectro de utilização do PRP vem se ampliando. São vários os relatos de uso na medicina, odontologia e veterinária. Como utiliza sangue do próprio paciente, é um produto seguro e o seu uso tem sido facilitado pela existência de dispositivos de preparo comercialmente disponíveis. O PRP, no entanto, não é um produto único. Variações nas preparações e concentrações dos componentes, a presença ou ausência de leucócitos, entre outros fatores, podem afetar os resultados dos estudos e comparação de resultados. $O$ conhecimento do mecanismo de ação, métodos de preparo e classificação ajuda os profissionais de saúde a melhor entender essa nova terapia.

\section{REFERÊNCIAS}

1. AHMAD Z, et al. The role of platelet rich plasma in musculoskeletal science. JRSM short reports. 2012; 3(6): 40.

2. ALI RA, et al. Platelets: essential components of the immune system. Current trends in immunology. 2015; 16: 6578.

3. ALVES R, GRIMALT R. A Review of Platelet-Rich Plasma: History, Biology, Mechanism of Action, and Classification. Skin Appendage Disorders. 2018; 4(1): 18-24.

4. ARAKI J, et al. Optimized Preparation Method of Platelet-Concentrated Plasma and Noncoagulating Platelet-Derived Factor Concentrates: Maximization of Platelet Concentration and Removal of Fibrinogen. Tissue Engineering Part C: Methods. 2012; 18(3): 176-185.

5. BARBER FA. Prp as an Adjunct to Rotator Cuff Tendon Repair. Sports Medicine and Arthroscopy Review. 2018; 26(2): 42-47.

6. BENNELL KL, et al. Platelet-Rich Plasma for the Management of Hip and Knee Osteoarthritis. Current Rheumatology Reports. 2017; 19(5): 24.

7. BHANOT S, ALEX JC. Current applications of platelet gels in facial plastic surgery. Facial Plast Surg. 2002; 18(1): 27-33.

8. BLAIR P, FLAUMENHAFT R. Platelet alpha-granules: basic biology and clinical correlates. Blood Reviews. 2009; 23(4): 177-189.

9. BORRIONE $\mathrm{P}$, et al. The use of platelet-rich plasma (PRP) in the treatment of gastrocnemius strains: a retrospective observational study. Platelets. 2018; 29(6): 596-601.

10. BOSWELL SG, et al. Platelet-Rich Plasma: A Milieu of Bioactive Factors. Arthroscopy: The Journal of Arthroscopic \& Related Surgery. 2012; 28(3): 429-439.

11. BRASIL. Ministério da Saúde. Secretaria de Atenção à Saúde. Departamento de Atenção Especializada e Temática. Caderno de informação: sangue e hemoderivados : dados de 2016 [recurso eletrônico] / Ministério da Saúde, Secretaria de Atenção à Saúde, Departamento de Atenção Especializada e Temática. - Brasília: Ministério da Saúde, 2018.

12. CALEY MP, et al. Metalloproteinases and Wound Healing. Advances in Wound Care. 2015; 4(4): $225-234$.

13. CAVALLO C, et al. Platelet-Rich Plasma: The Choice of Activation Method Affects the Release of Bioactive Molecules. BioMed Research International. Volume 2016, Article ID 6591717, 7 pages, 2016. 
14. CHARLES MD, et al. The Role of Biologic Therapy in Rotator Cuff Tears and Repairs. Current Reviews in Musculoskeletal Medicine. 2018; 11(1): 150-161.

15. CHUNG R, et al. Potential roles of growth factor PDGF-BB in the bony repair of injured growth plate. Bone. 2009; 44(5): 878-885.

16. CHICHARRO-ALCÁNTARA D, et al. Platelet Rich Plasma: New Insights for Cutaneous Wound Healing Management. Journal of Functional Biomaterials. 2018; 9(1).

17. CIESLIK-BIELECKA A, et al. Microbidical properties of leucocyte- and platelet rich plasma/fibrin (L-PRP/L-PRF): new perspectives. Journal of Biological Regulators \& Homeostatic Agents. 2012; 26(2)(S): 0-0.

18. DAVIS VL, et al. Platelet-Rich Preparations to Improve Healing. Part II: Platelet Activation and Enrichment, Leukocyte Inclusion, and Other Selection Criteria. Journal of Oral Implantology. 2014; 40(4): 511-521.

19. DELONG JM, et al. Platelet-Rich Plasma: The PAW Classification System. Arthroscopy: The Journal of Arthroscopic \& Related Surgery. 2012; 28(7): 998-1009.

20. DHURAT R, SUKESH M. Principles and methods of preparation of platelet-rich plasma: A review and author's perspective. Journal of Cutaneous and Aesthetic Surgery. 2014; 7(4): 189.

21. EHRENFEST DMD, et al. Classification of platelet concentrates: from pure platelet-rich plasma (P-PRP) to leucocyteand platelet-rich fibrin (L-PRF). Trends in Biotechnology. 2009; 27(3): 158-167.

22. FOLLONIER CASTELLA L, et al. Regulation of myofibroblast activities: calcium pulls some strings behind the scene. Experimental Cell Research. 2010; 316(15): 2390-2401.

23. GAUDRIC A, et al. Autologous platelet concentrate for the treatment of full-thickness macular holes. Graefe's Archive for Clinical and Experimental Ophthalmology. 1995; 233(9): 549-554.

24. KNOP E, et al. Plasma rico em plaquetas no tratamento da osteoartrite. Revista Brasileira de Reumatologia. 2016; 56(2): 152-164.

25. KOH TJ, DIPIETRO LA. Inflammation and wound healing: the role of the macrophage. 2011; 13: e23.

26. LACCI KM, DARDIK A. Platelet-Rich Plasma: Support for Its Use in Wound Healing. Yale Journal of Biology and Medicine 83. 2010; 1-9.

27. LANA JFSD, et al. Contributions for classification of platelet rich plasma - proposal of a new classification: MARSPILL. Regenerative Medicine. 2017; 12(5): 565-574.

28. MEHTA SK, et al. Effect of platelet dense granule contents upon osteoblast viability. Biomedical Sciences Instrumentation. 2012; 48: 288-295.

29. MENCHISHEVA Y, et al. Use of platelet-rich plasma to facilitate wound healing. International Wound Journal, 15 nov. 2018.

30. MENG R, et al. Defective release of a granule and lysosome contents from platelets in mouse Hermansky-Pudlak syndrome models. Blood. 2015; 125(10): 1623-1632.

31. MOOJEN DJF, et al. Antimicrobial activity of platelet-leukocyte gel against Staphylococcus aureus. Journal of Orthopaedic Research. 2008; 26(3): 404-410.

32. PIERCE GF, et al. Role of platelet-derived growth factor in wound healing. Journal of Cellular Biochemistry. 1991; 45(4): 319-326.

33. REINKE JM, SORG H. Wound Repair and Regeneration. European Surgical Research. 2012; 49(1): 35-43.

34. SCHMIDT MB, et al. A review of the effects of insulin-like growth factor and platelet derived growth factor on in vivo cartilage healing and repair. Osteoarthritis and Cartilage. 2006; 14(5): 403-412.

35. SHEN L, et al. The temporal effect of platelet-rich plasma on pain and physical function in the treatment of knee osteoarthritis: systematic review and meta-analysis of randomized controlled trials. Journal of Orthopaedic Surgery and Research. 2017; 12(1): 16.

36. STÄHLI A, et al. The use of platelet-rich plasma to enhance the outcomes of implant therapy: A systematic review. Clinical Oral Implants Research. 2018; 29(Suppl 18): 20-36.

37. THIBAULT MM, et al. Fibronectin, vitronectin, and collagen I induce chemotaxis and haptotaxis of human and rabbit mesenchymal stem cells in a standardized transmembrane assay. Stem Cells and Development. 2007; 16(3): 489502.

38. WHITMAN DH, et al. Platelet gel: An autologous alternative to fibrin glue with applications in oral and maxillofacial surgery. Journal of Oral and Maxillofacial Surgery. 1997; 55(11): 1294-1299.

39. XU Z, et al. Comparative evaluation of leukocyte- and platelet-rich plasma and pure platelet-rich plasma for cartilage regeneration. Scientific Reports. 2017; 7: 43301.

40. ZHOU Y, et al. The differential effects of leukocyte-containing and pure platelet-rich plasma (PRP) on tendon stem/progenitor cells - implications of PRP application for the clinical treatment of tendon injuries. Stem Cell Research \& Therapy. 2015; 6(1). 